\title{
ACCRETION-DISK CORONA ADVECTED BY EXTERNAL RADIATION DRAG
}

\author{
Y.WATANABE AND J.FUKUE \\ Osaka Kyoiku University
}

\section{Motivation}

Accretion-disk corona (ADC) is required from observational as well as theoretical reasons. In almost all of traditional studies, however, a stationary corona has been assumed; i.e., the corona gas corotates with the underlying (Keplerian) accretion disk, and the radial motion is ignored. Recently, in the theory of accretion disks a radiative interaction between the gas and the external radiation field has attracted the attention of researchers. In particular the radiation drag between the gas and the external radiation field becomes important from the viewpoint of the angular-momentum removal. We thus examine the effect of radiation drag on the accretion-disk corona above/below the accretion disk (Watanabe, Fukue 1996a, b). We suppose that an accretion disk can be described by the standard disk, and that radiation fields are produced by the central luminous source and the accretion disk, itself. In general an accretion-disk corona under the influence of strong radiation fields dynamically infalls (advected) toward the center.

\section{Advection Disk Corona}

Let us suppose an axisymmetric disk corona above/below a standard accretion disk, which is steadily rotating around a central object (Fig.1). We ignore the self-gravity of the corona gas and disk gas. The disk corona is assumed to be geometrically thin, and thus, the physical quantities are integrated over the vertical direction. It is also assumed to be effectively optically thin. When the central source becomes luminous, the angular momentum is removed through the action of radiation drag from the central 
source radiation, and the advection/infall of the corona gas is remarkably enhanced. When the accretion disk is luminous, the corona gas tends to corotate with the disk and the radial infall of the corona gas is suppressed. When the corona gas is hot, the pressure-gradient force supports the radial hydrostatic-equilibrium of the corona and the radial infall of the corona gas is suppressed.
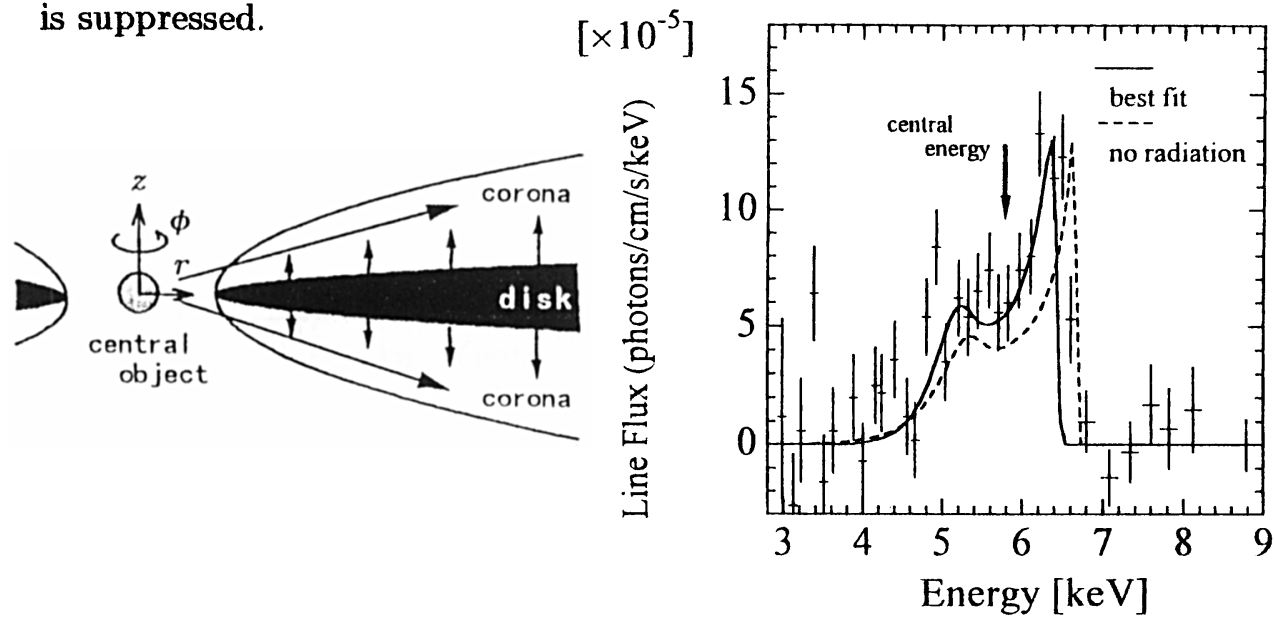

Fig. 1. (Left) Model. Fig. 2. (Right) Best-fit line profile of the present model to the Xray emission line detected in MCG-6-30-15. The crosses are the observed X-ray lines reproduced from Tanaka et al. (1995).

\section{Line Profiles}

The line profiles from advection disk coronae generally have double-peaked features. When the central object becomes more luminous, the rotation velocity of the disk corona is smaller, and therefore, the redshift becomes smaller and the separation of two peaks is smaller. The profiles do not depend very much on the disk luminosity. In the relativistic case, the blue peaks become more intense than the red peaks due mainly to the beaming effect, while the centroid energy is smaller than in the non-relativistic case, mainly due to the gravitational redshift.

We fit the X-ray line spectrum of MCG-6-30-15 (Tanaka et al. 1995) by the present model (Fig.2). This means that if the X-ray lines from MCG6-30-15 are emitted from the corona above the accretion disk, the corona may be strongly affected by the radiation drag, and infall dynamically to the central black hole.

\section{References}

Tanaka Y. et al. 1995, Nature 375, 659

Watanabe Y., Fukue J. 1996a, PASJ 48, 841

Watanabe Y., Fukue J. 1996b, PASJ 48, 849 